\title{
Effect of increasing nickel and molybdenum content in austenitic steel on thermal deformation conditions for achieving the peak strain
}

\author{
A.S. Tsvetkov ${ }^{\dagger, 1,2}$, I. V. Teplukhina ${ }^{1}$, A. V. Kosulnikova ${ }^{1}$ \\ †a.s.tsvetkow@gmail.com

\begin{abstract}
${ }^{1}$ NRC “Kurchatov institute” - CRISM “Prometey”, St. Petersburg, 191015, Russia
${ }^{2}$ Peter the Great St. Petersburg Polytechnic University, St. Petersburg, 195251, Russia
\end{abstract}

\begin{abstract}
Obtaining the required grain size in steel forgings with austenitic metal structure is a problem, especially for the forged thick-walled blanks made by forging on hydraulic presses. For such steels, there is no possibility of grain refinement during heat treatment like for steels of the bainitic and martensitic classes which undergo polymorphic transformation. In this case, refinement to the required grain size is possible only at the stage of hot plastic deformation as a result of recrystallization. Formation of required grain size happens in the process of forging and this grain size is one of the control parameters when blanks acceptance is carried out. The rate of nucleation of new grains increases sharply during dynamic recrystallization at the peak strain $\left(e_{\mathrm{p}}\right)$ and depends on temperature, deformation rate, initial grain size and chemical composition. The article presents the results of measurements of $e_{\mathrm{p}}$ and peak stress $\left(\sigma_{\mathrm{p}}\right)$ in austenitic steels with a nickel content of $\approx 10,20,25 \%$ and a molybdenum content of $\approx 0.01,2.5 \%$ under thermodeformation conditions typical for forging large-sized blanks on a hydraulic press. It is shown that an increase in the nickel content up to $25 \%$ in combination with alloying with molybdenum, in the temperature range $900-1200^{\circ} \mathrm{C}$, has the most significant effect on the level of $e_{\mathrm{p}}$ and $\sigma_{\mathrm{p}}$ values during forging at the $10^{-1} \mathrm{~s}^{-1}$ deformation rate. At deformation rates of $10^{-2}$ and $10^{-3} \mathrm{~s}^{-1}$, the values of $e_{\mathrm{p}}$ and $\sigma_{\mathrm{p}}$ of the studied steel grades are practically the same.
\end{abstract}

Keywords: core baffle, austenitic steel, deformation resistance, recrystallization, deformation rate, peak strain, peak stress.

УДК: 669.01:620.173.251.2

\section{Влияние увеличения содержания никеля и молибдена в аустенитной стали на термодеформационные условия достижения пороговой степени деформации}

\author{
Цветков А. С. ${ }^{\dagger, 1,2}$, Теплухина И. В. ${ }^{1}$, Косульникова А. В. ${ }^{1}$ \\ ${ }^{1}$ НИЦ «Курчатовский институт»- ЦНИИ КМ «Прометей», С.-Петербург, 191015, Россия \\ ${ }^{2}$ Политехнический университет Петра Великого, С.-Петербург, 195251, Россия
}

Получение требуемого размера зерна в заготовках из стали, имеющей аустенитную структуру, представляет собой определенную проблему, особенно для толстостенных заготовок, изготавливаемых ковкой на гидравлических прессах. Для аустенитных сталей отсутствует возможность измельчения зерна при проведении основной термической обработки, как для сталей бейнитного и мартенситного классов, претерпевающих при термической обработке полиморфные превращения. Для этих сталей единственной возможностью получения требуемого размера зерна в металле заготовки является использование возможности измельчения зерна за счет рекристаллизации при ковке. Именно на операциях ковки формируется зеренная структура металла, являющаяся одним из параметров, подлежащих контролю при проведении сдаточных испытаний заготовок. Скорость зарождения новых зерен при динамической рекристаллизации резко возрастает с увеличением степени деформации выше пороговой $\left(e_{\mathrm{p}}\right)$ и зависит от температуры, скорости деформации, исходного размера зерна и легирования. В статье представлены результаты определения значений $e_{\mathrm{p}}$ и пороговых напряжений $\left(\sigma_{\mathrm{p}}\right)$ в аустенитных сталях с варьированием содержания никеля $\approx 10,20,25 \%$ и молибдена $\approx 0.01,2.5 \%$ при термодеформационных условиях, характерных 
для ковки крупногабаритных заготовок на гидравлическом прессе. Показано, что увеличение содержания никеля до $\approx 25 \%$ в совокупности с легированием молибденом, в температурном интервале $900-1200^{\circ} \mathrm{C}$ оказывает наиболее существенное влияние на уровень $e_{\mathrm{p}}$ и $\sigma_{\mathrm{p}}$ при деформировании со скоростью $10^{-1} \mathrm{c}^{-1}$. При скоростях деформирования $10^{-2}$ и $10^{-3} \mathrm{c}^{-1}$ значения $e_{\mathrm{p}}$ и $\sigma_{\mathrm{p}}$ исследованных марок стали практически совпадают.

Ключевые слова: внутрикорпусная выгородка, аустенитная сталь, сопротивление деформации, рекристаллизация, скорость деформирования, пороговая степень деформации, пороговые напряжения.

\section{1. Введение}

В настоящее время в России ведется создание водоводяных энергетических реакторов (ВВЭР) повышенной мощности со сроком службы не менее 60 лет [1]. Внутрикорпусные устройства (ВКУ) служат для размещения и фиксации активной зоны реактора, организации движения теплоносителя и охлаждения активной зоны, размещения и обеспечения движения органов системы управления и защиты, уменьшения облучения корпуса реактора. В состав ВКУ входят шахта внутрикорпусная, блок защитных труб и выгородка. В качестве конструкционного материала ВКУ ВВЭР применяется аустенитная сталь марки 08X18Н10Т. При обосновании срока службы ВКУ ВВЭР критическим элементом с точки зрения определения срока их эксплуатации является выгородка, испытывающая наибольшие радиационные нагрузки и имеющая максимальную температуру вследствие $\gamma$-разогрева в результате облучения [2].

В работах [3 - 5] показано, что работоспособность за пределами проектного срока службы внутрикорпусной выгородки ВВЭР новых проектов (ВВЭР-1200, ВВЭР-ТОИ) не может быть гарантирована при консервативном прогнозе радиационного распухания в случае, если она будет изготовлена из использующейся в настоящий момент аустенитной стали марки 08Х18Н10Т.

Основными причинами нарушения работоспособности выгородки за пределами срока службы являются достижение критического формоизменения в результате радиационного распухания, а также потеря пластичности и трещиностойкости материала при резком снижении прочности $[4,6]$. При высоком уровне распухания $(S>10-15 \%)$ происходит охрупчивание материала до пластичности, близкой к нулевой, и падение прочности. Формоизменение выгородки ограничивает ресурс ВКУ, т.к. при протекании этого процесса может произойти контакт выгородки с тепловыделяющими сборками (ТВC) и в результате может быть затруднено извлечение ТВС из реактора $[4,5]$. Кроме того, контакт выгородки с шахтой может привести к перераспределению потоков теплоносителя и, как следствие, к нарушению теплосъема с активной зоны.

Таким образом, воздействие повреждающих факторов при эксплуатации на материал ВКУ вызывает сильную деградацию его свойств, а также приводит к возникновению внутренних напряжений. Сочетание низких свойств материала и напряжений может привести к преждевременному разрушению элементов ВКУ и потере их работоспособности.

Для обеспечения возможности создания ВВЭР с более высокой мощностью и гарантированной работо- способностью выгородки за пределами проектного срока службы, необходима корректировка легирующей композиции применяемой стали для ВКУ с целью ослабления механизмов деградации материала под влиянием облучения и рабочей температуры. В связи с вышесказанным, в настоящее время разрабатывается новая радиационно-стойкая аустенитная сталь [2], содержащая в своем составе:

- никель в количестве до 20-25\% для уменьшения склонности стали к радиационному распуханию и исключения возможности $\gamma \rightarrow \alpha$-превращения вследствие обеднения матрицы никелем в результате облучения;

- молибден в количестве до 2-3\% для уменьшения степени сегрегационных процессов легирующих и примесных элементов в матрице в результате воздействия облучения и рабочей температуры.

Одним из контролируемых параметров для металла заготовок ВКУ является размер зерна, который должен быть не крупнее G3 по шкале 3 ГОСТ 5639. Необходимо отметить, что для аустенитных сталей отсутствует возможность измельчения зерна при проведении основной термической обработки [7], как для сталей бейнитного и мартенситного классов, претерпевающих при термической обработке полиморфные превращения. В этом случае единственной возможностью получения требуемого размера зерна в металле заготовки является использование возможности измельчения зерна при рекристаллизации при ковке [8].

Существующая в настоящее время технология ковки заготовок из стали марки 08X18Н10Т обеспечивает получение требуемого размера зерна в заготовках для ВКУ, в том числе и в наиболее толстостенных заготовках для колец выгородки. Корректировка легирующей композиции применяемой стали (увеличение содержания никеля до 20-25\%, а также легирование молибденом) требует уточнения технологических параметров горячей деформации с целью обеспечения условий протекания динамической рекристаллизации при ковке заготовки.

Основной интерес в этом случае представляет определение термодеформационных условий достижения пороговой степени деформации $\left(e_{\mathrm{p}}\right)$, отмечающей прохождение динамической рекристаллизации, при которой начинается зарождение новых зерен. Кроме того, при значительном увеличении в стали содержания никеля (более чем в два раза), следует ожидать повышения сопротивления пластической деформации, что, возможно, потребует использования при ковке заготовок из новой стали более мощного прессового оборудования.

В связи с вышесказанным, исследование влияния увеличения содержания никеля и легирования молибденом в аустенитной стали на сопротивление дефор- 
мированию и достижение $e_{\mathrm{p}}$ в температурном и скоростном интервале ковки крупногабаритных заготовок на гидравлическом прессе является актуальным и представляет научный и практический интерес.

\section{2. Материал для исследования и методика испытаний}

Исследование проводилось на металле опытных плавок, химический состав которых по основным легирующим элементам приведен в Табл. 1. Образцы для исследований отбирались из полос, откованных из опытных слитков массой 500 кг свободной ковкой на молотах по одной технологии.

Для определения $e_{\mathrm{p}}$ и пороговых значений сопротивления деформации $\left(\sigma_{\mathrm{p}}\right)$ для исследуемых материалов была проведена серия экспериментов на однократное изотермическое сжатие образцов диаметром 10 мм и высотой 15 мм со скоростями деформирования $10^{-1}$, $10^{-2}, 10^{-3} \mathrm{c}^{-1}$ при температурах $900,1000,1100,1200^{\circ} \mathrm{C}$ на термодеформационном комплексе Gleeble-3800. Деформирование образцов осуществлялось до истинной степени деформации $e=0.69$.

Выбор температур деформирования связан с распределением температурного поля по толщине крупногабаритной поковки в процессе каждого выноса (периода нахождения поковки на прессе в интервале ковочных температур). Степень деформации при проведении испытаний определена с учетом имеющихся данных по градиенту $e$ по сечению заготовки при каждом единичном обжатии бойка на этапе раскатки при ковке заготовки кольца выгородки. Интервал скоростей деформирования обусловлен тем, что фактические скорости деформирования при свободной ковке на гидравлическом прессе также варьируются в значительных пределах по толщине заготовки и зависят от удаленности слоя металла от очага деформации. В соответствии с [9-11] при ковке на гидравлических прессах скорости деформирования на основных операциях составляют $\sim 10^{-1}-10^{-3} \mathrm{c}^{-1}$.

Перед деформированием образцы нагревали до $1200^{\circ} \mathrm{C}$ со скоростью $5^{\circ} \mathrm{C} / \mathrm{c}$ прямым пропусканием тока в условиях низкого вакуума. После изотермической выдержки в течение 30 с проводилось охлаждение образцов до температуры деформирования с последующей выдержкой в течение 10 с. После окончания деформирования осуществлялось ускоренное охлаждение образцов сжатым воздухом.
По полученным диаграммам деформирования определялись значения $e_{\mathrm{p}}$ и $\sigma_{\mathrm{p}}$, соответствующие началу развития динамической рекристаллизации.

\section{3. Результаты исследований и их обсуждение}

Влияние температуры и скорости деформирования на $e_{\mathrm{p}}$ и $\sigma_{\mathrm{p}}$ исследованных сталей представлено на Рис. 1 и 2. Полученные результаты подтвердили известные зависимости влияния скорости и температуры деформирования на $e_{\mathrm{p}}$ и $\sigma_{\mathrm{p}}[8,10,12]$ для всех исследованных составов - уровень значений $e_{\mathrm{p}}$ и $\sigma_{\mathrm{p}}$ снижается с ростом температуры и уменьшением скорости деформирования металла.

Как видно из полученных результатов, для опытных составов 1 и 2 при исследованных температурах и скоростях деформирования значения $e_{\mathrm{p}}$ и $\sigma_{\mathrm{p}}$ практически одинаковы, то есть увеличение содержания никеля в опытной стали с 20 до 25\% при одинаковом содержании молибдена не влияет на значения $e_{\mathrm{p}}$ и $\sigma_{\mathrm{p}}$.

Увеличение содержания никеля с $10 \%$ в применяемой стали до $\approx 20-25 \%$ в опытных составах, а также снижение температуры и повышение скорости деформирования приводят к увеличению значений $\sigma_{\text {p }}$. Упрочнение стали выше при деформировании в низкотемпературной области исследованного температурного интервала. Максимальный уровень значений $\sigma$ фиксируется при скорости деформирования $10^{-1} \mathrm{c}^{-1}$ для опытных составов 1 и 2 (Рис. 1a). При этом различие в значениях $\sigma_{\text {p }}$ для опытных составов и стали марки $08 \mathrm{X} 18 \mathrm{H} 10 \mathrm{~T}$ при температуре $1000^{\circ} \mathrm{C}$ составляет около $60 \mathrm{MПа,} \mathrm{при} \mathrm{температуре} 1100^{\circ} \mathrm{C} \approx 30 \mathrm{MПа}$, а при температуре $1200^{\circ} \mathrm{C}$ различие отсутствует. Различие в значениях $\sigma_{\text {p }}$ для опытных составов и стали марки 08X18Н10Т при температурах деформирования $900-1200^{\circ} \mathrm{C}$ и скоростях $10^{-2}$ и $10^{-3} \mathrm{c}^{-1}$ незначительно (Рис. 1b,c). Полученные результаты свидетельствуют, что для ковки сталей новых легирующих композиций возможно использование применяемого прессового оборудования (гидравлического пресса 12000 тс) при условии обеспечения необходимых усилий.

Из данных, представленных на Рис. 2, видно, что в стали марки 08Х18Н10Т при скоростях $10^{-1}-10^{-3} \mathrm{c}^{-1}$ и температуре деформирования $900^{\circ} \mathrm{C}$ достижение $e_{\mathrm{p}}$ при деформировании до степени $e=0.69$ не фиксируется. В опытных составах 1 и 2 достижение $e_{\mathrm{p}}$ отмечено во всем исследованном температурно-скоростном диапа-

Табл. 1. Химический состав исследуемых марок аустенитной стали.

Table 1. Chemical composition of studied austenitic steel.

\begin{tabular}{|c|c|c|c|c|c|}
\hline \multirow{2}{*}{$\begin{array}{c}\text { Исследуемые марки стали } \\
\text { Studied steel grades }\end{array}$} & \multicolumn{5}{|c|}{$\begin{array}{c}\text { Химический состав, масс.\% } \\
\text { Chemical composition, mass.\% }\end{array}$} \\
\cline { 2 - 6 } & $\mathrm{C}$ & $\mathrm{Cr}$ & $\mathrm{Ni}$ & $\mathrm{Mo}$ & $\mathrm{Ti}$ \\
\hline $\begin{array}{c}\text { Используемая сталь - 08X18H10T } \\
\text { Used steel - 08Кh18N10T }\end{array}$ & 0.071 & 17.5 & 10.2 & 0.01 & 0.5 \\
\hline $\begin{array}{c}\text { Опытный состав 1 - сталь 08X16H20M2T } \\
\text { Studied steel 1 - 08Кh16N20M2T }\end{array}$ & 0.065 & 15.3 & 20.2 & 2.59 & 0.6 \\
\hline $\begin{array}{c}\text { Опытный состав 2 - сталь 10Х16H25M2T } \\
\text { Studied steel 2 - 10Кh16N25M2T }\end{array}$ & 0.081 & 15.5 & 24.3 & 2.49 & 0.7 \\
\hline
\end{tabular}




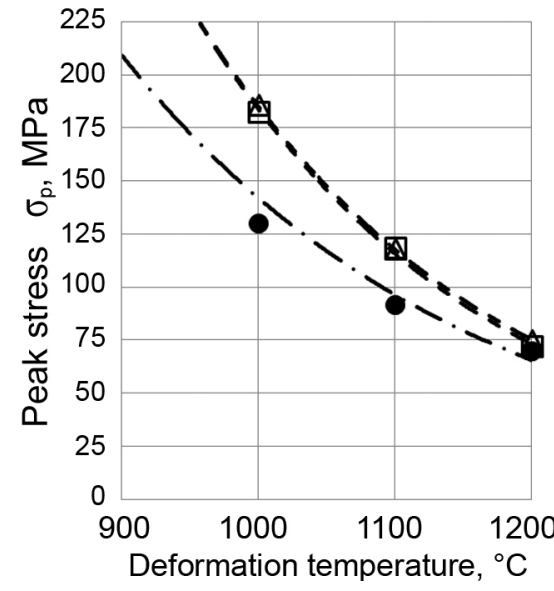

a

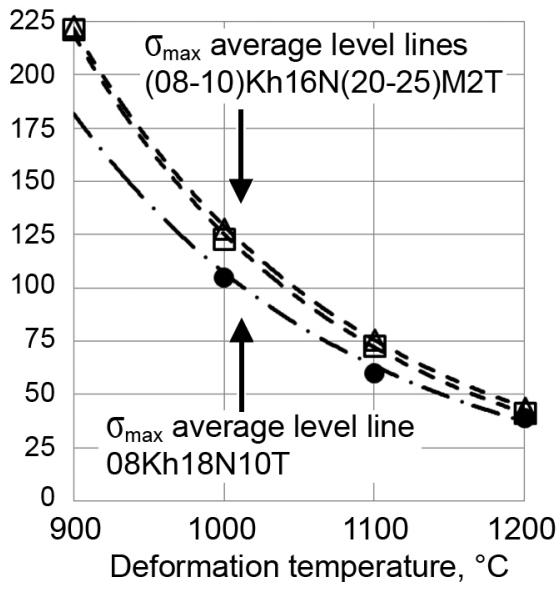

b

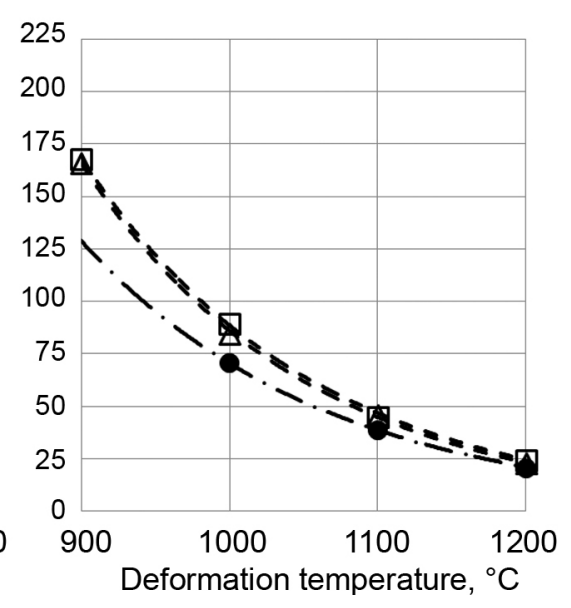

C

Рис. 1. Влияние температуры $\left(900-1200^{\circ} \mathrm{C}\right)$ и скорости деформирования: $10^{-1} \mathrm{c}^{-1}$ (a), $10^{-2} \mathrm{c}^{-1}$ (b), $10^{-3} \mathrm{c}^{-1}$ (c) на изменение $\sigma_{\mathrm{p}}$. Экспериментальные значения $\sigma_{\mathrm{p}}: \bullet-$ steel 08X18H10T (содержание Ni/Mo, масс.\% 10/0.01); $\square-08 \mathrm{X} 16 \mathrm{H} 20 \mathrm{M} 2 \mathrm{~T}$ (содержание $\mathrm{Ni} / \mathrm{Mo}$, масс.\% 20/2.5); $\triangle-10 \mathrm{X} 16 \mathrm{H} 25 \mathrm{M} 2 \mathrm{~T}$ (содержание Ni/Mo, масс.\% 25/2.5).

Fig. 1. The influence of deformation temperature $\left(900-1200^{\circ} \mathrm{C}\right)$ and ration: $10^{-1} \mathrm{~s}^{-1}$ (a), $10^{-2} \mathrm{~s}^{-1}$ (b), $10^{-3} \mathrm{~s}^{-1}$ (c) on the $\sigma_{\mathrm{p}}$ changing. $\sigma$ experimental values: $\bullet$ - steel 08Kh18N10T (Ni/Mo content, mass.\% 10/0.01); $\square-08 \mathrm{Kh} 16 \mathrm{~N} 20 \mathrm{M} 2 \mathrm{~T}$ (Ni/Mo content, mass. $\% \sim 20 / 2.5$ ); $\triangle \triangle^{\mathrm{p}}-10 \mathrm{Kh} 16 \mathrm{~N} 25 \mathrm{M} 2 \mathrm{~T}(\mathrm{Ni} / \mathrm{Mo}$ content, mass.\% 25/2.5).

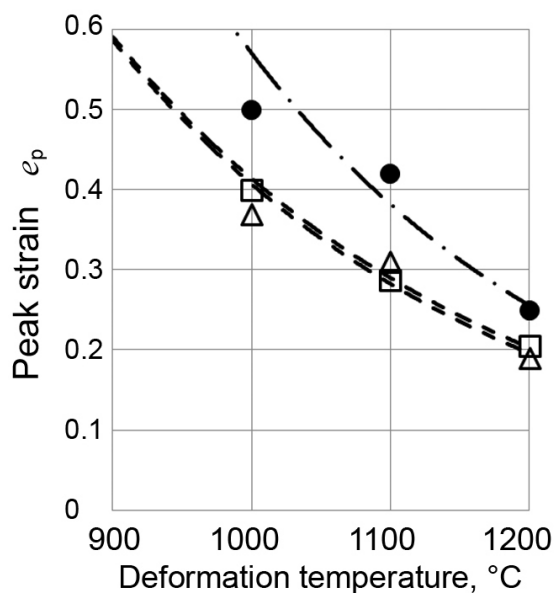

a

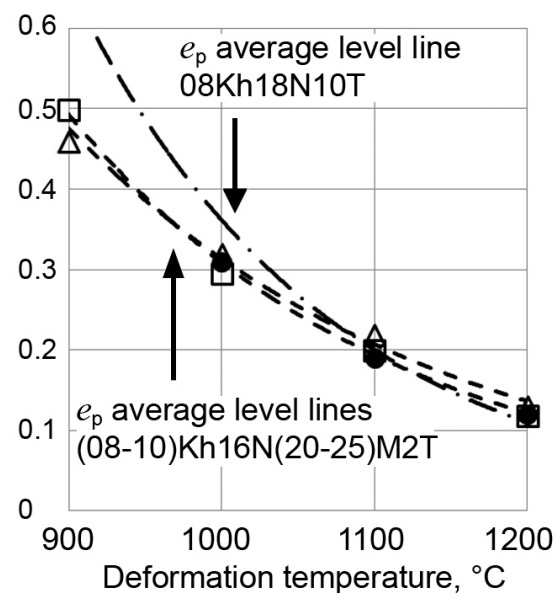

b

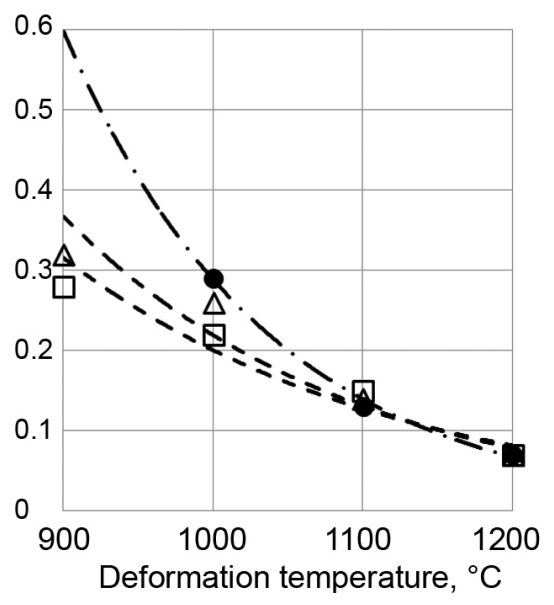

C

Рис. 2. Влияние температуры $\left(900-1200^{\circ} \mathrm{C}\right)$ и скорости деформирования: $10^{-1} \mathrm{c}^{-1}$ (a), $10^{-2} \mathrm{c}^{-1}$ (b), $10^{-3} \mathrm{c}^{-1}$ (c) на достижение $e_{\mathrm{p}}$. Экспериментальные значения $e_{\mathrm{p}}$ : — steel 08X18H10T (содержание Ni/Mo, масс.\% 10/0.01); $\square-08 \mathrm{X} 16 \mathrm{H} 20 \mathrm{M} 2 \mathrm{~T}$ (содержание $\mathrm{Ni} / \mathrm{Mo}$, масс.\% 20/2.5); $\triangle-10 \mathrm{X} 16 \mathrm{H} 25 \mathrm{M} 2 \mathrm{~T}$ (содержание Ni/Mo, масс.\% 25/2.5).

Fig. 2. The influence of deformation temperature $\left(900-1200^{\circ} \mathrm{C}\right)$ and ration: $10^{-1} \mathrm{~s}^{-1}(\mathrm{a}), 10^{-2} \mathrm{~s}^{-1}(\mathrm{~b}), 10^{-3} \mathrm{~s}^{-1}(\mathrm{c})$ on the $e_{\mathrm{p}}$ achieving. $e_{\mathrm{p}}$ experimental values: $\bullet$ steel $08 \mathrm{Kh} 18 \mathrm{~N} 10 \mathrm{~T}(\mathrm{Ni} / \mathrm{Mo}$ content, mass. $\% \sim 10 / 0.01)$; $\square-08 \mathrm{Kh} 16 \mathrm{~N} 20 \mathrm{M} 2 \mathrm{~T}(\mathrm{Ni} / \mathrm{Mo}$ content, mass. $\% \sim 20 / 2.5)$; $\triangle-10 \mathrm{Kh} 16 \mathrm{~N} 25 \mathrm{M} 2 \mathrm{~T}(\mathrm{Ni} / \mathrm{Mo}$ content, mass. $\% \sim 25 / 2.5)$.

зоне, кроме деформирования при скорости $10^{-1} \mathrm{c}^{-1}$ при $900^{\circ} \mathrm{C}$ (Рис. $\left.2 \mathrm{a}\right)$.

Следует отметить, что при раскатке заготовки кольца выгородки ее поверхностные слои могут охлаждаться до температуры $900-950^{\circ} \mathrm{C}$. С учетом полученных данных измельчение зерна за счет динамической рекристаллизации в поверхностных слоях заготовки из стали марки $08 \mathrm{X} 18 \mathrm{H} 10 \mathrm{~T}$ ( $e_{\mathrm{p}}$ при $900^{\circ} \mathrm{C}$ не достигается) невозможно, в то время как в сталях опытного состава наблюдается достижение $e_{\mathrm{p}}$ при скоростях деформирования $10^{-2}-10^{-3} \mathrm{c}^{-1}$ при этих температурах.

Наибольшее влияние легирования никелем и молибденом опытных составов на достижение значений $e_{\mathrm{p}}$ во всем исследованном температурном интервале происходит при скорости деформирования $10^{-1} \mathrm{c}^{-1}$ (Рис. 2 а). При скоростях деформирования $10^{-2}$ и $10^{-3} \mathrm{c}^{-1}$ при температуре $900^{\circ} \mathrm{C}$ также отмечено снижение уровня значений $e_{\mathrm{p}}$. В интервале температур деформирования $1000-1200^{\circ} \mathrm{C}$ при данных скоростях для опытных составов и применяемой стали значения $e_{\mathrm{p}}$ практически совпадают (Рис. 2 b, с).

С учетом полученных результатов можно сделать заключение, что опытные стали, содержащие 20-25\% никеля и 2.5\% молибдена, будут более технологичны с точки зрения измельчения зерна при раскатке за счет протекания динамической рекристаллизации 
во всем объеме поковки, чем применяемая сталь марки 08X18Н10T, в которой протекание динамической рекристаллизации в поверхностных слоях проблематично.

\section{4. Заключение}

В стали марки 08X18Н10Т при температуре деформации $900^{\circ} \mathrm{C}$ достижение $e_{\mathrm{p}}$ при деформировании до степени $e=0.69$ не зафиксировано при всех исследованных скоростях деформации. В новых сталях опытного состава достижение $e_{\mathrm{p}}$ отмечено во всем исследованном температурно-скоростном диапазоне, кроме деформирования при скорости деформации $10^{-1} \mathrm{c}^{-1}$ при $900^{\circ} \mathrm{C}$.

Наибольшее влияние увеличения содержания никеля и молибдена на значения $e_{\mathrm{p}}$ и $\sigma_{\mathrm{p}}$ происходит при скорости деформации $10^{-1} \mathrm{c}^{-1}$ во всем исследованном температурном интервале. При скоростях деформирования $10^{-2}$ и $10^{-3} \mathrm{c}^{-1}$ в интервале температур деформации $1000-1200^{\circ} \mathrm{C}$ для опытных составов и применяемой стали значения $e_{\mathrm{p}}$ и $\sigma_{\mathrm{p}}$ практически совпадают.

Таким образом, увеличение содержание никеля до 20-25\% и легирование молибденом увеличивает склонность стали к динамической рекристаллизации при скоростях деформирования $10^{-1}-10^{-3} \mathrm{c}^{-1}$. При этом для применяемого прессового оборудования увеличение уровня значений $\sigma$ незначительно во всем исследованном интервале скоростей и температур деформации.

\section{Литература/References}

1. G. P. Karzov, B.Z. Margolin, I. V. Teplukhina et al. Atomic energy. 1 (121), 25 (2016). (in Russian) [Г.П. Карзов, Б.3. Марголин, И.В. Теплухина и др. Атомная энергия. 1 (121), 25 (2016).]

2. I. P. Kursevich, G. P. Karzov, B. Z. Margolin et al. Problems of material science. 3 (71), 146 (2012). (in Russian)
[И.П. Курсевич, Г.П. Карзов, Б.З. Марголин и др. Вопросы материаловедения. 3 (71), 146 (2012).]

3. V.M. Makhin, V.I. Tsofin, V.M. Komolov et al. Nuclear science and technology issues. 25, 135 (2009). (in Russian) [В. М. Махин, В.И. Цофин, В. М. Комолов и др. Вопросы атомной науки и техники. 25, 135 (2009).]

4. G.P. Karzov, B.Z. Margolin. Rosenergoatom. 2, 8 (2015). (in Russian) [Г.П. Карзов, Б.3. Марголин. Росэнергоатом. 2, 8 (2015).]

5. V.A. Piminov, V. V. Evdokimenko. Rosenergoatom. 2, 16 (2015). (in Russian) [В.А.Пиминов, В. В. Евдокименко. Росэнергоатом. 2, 16 (2015).]

6. V.S. Neustroev, F. A. Garner. Journal of nuclear materials. $386-388,157$ (2009). Crossref

7. I.V. Teplukhina, A.S. Tsvetkov, A.V. Kosulnikova. Letters on Materials. 10 (2), 179 (2020). (in Russian) [И. В. Теплухина, А. С. Цветков, А. В. Косульникова. Письма о материалах. 10 (2), 179 (2020).] Crossref

8. A.S. Tsvetkov, T.V. Knyazyuk, I. V. Teplukhina et al. Metallurgy of mechanical engineering. 5, 9 (2020). (in Russian) [А. С. Цветков, Т. В. Князюк, И.В. Теплухина и др. Металлургия машиностроения. 5, 9 (2020).]

9. A.K. Onishenko. Forging and stamping production. Metal forming. 4, 9 (2009). (in Russian) [А. К. Онищенко. Кузнечно-штамповочное производство. Обработка металлов давлением. 4, 9 (2009).]

10. M. V. Dobrynina, G. N. Filimonov, V. N. Pavlov. Problems of material science. 3 (67), 19 (2011). (in Russian) [М.В. Добрынина, Г.Н. Филимонов, В.Н. Павлов. Вопросы материаловедения. 3 (67), 19 (2011).]

11. A. Yu. Borisenko. Fundamental and applied problems of ferrous metallurgy. 28, 247 (2014). (in Russian) [А.Ю. Борисенко. Фундаментальные и прикладные проблемы черной металлургии. 28, 247 (2014).]

12. A. Dehghan-Manshadi, M.R. Barnett, P.D. Hodgson. Materials science and engineering A. 485, 664 (2008). $\underline{\text { Crossref }}$ 